\title{
Conscience périphérique et travail coopératif dans un café-restaurant
}

Awareness and cooperative work in a café-restaurant

Consciencia periférica y trabajo cooperativo en un café-restaurante

\section{Béatrice Cahour et Barbara Pentimalli}

\section{(Q) OpenEdition}

Journals

Édition électronique

URL : http://journals.openedition.org/activites/1578

DOI : 10.4000/activites. 1578

ISSN : 1765-2723

Éditeur

ARPACT - Association Recherches et Pratiques sur les ACTivités

\section{Référence électronique}

Béatrice Cahour et Barbara Pentimalli, « Conscience périphérique et travail coopératif dans un caférestaurant », Activités [En ligne], 2-1 | avril 2005, mis en ligne le 02 avril 2005, consulté le 21 avril 2019 URL : http://journals.openedition.org/activites/1578; DOI : 10.4000/activites.1578

\section{(c) (i) (2)}

Activités est mis à disposition selon les termes de la licence Creative Commons Attribution - Pas d'Utilisation Commerciale - Pas de Modification 4.0 International. 


\title{
Conscience périphérique et travail coopératif dans un café-restaurant
}

\author{
Béatrice Cahour \\ C.N.R.S. IRIT-GRIC, 118 route de Narbonne, 31062 Toulouse, France \\ bcahour@irit.fr
}

Barbara Pentimalli

Institut Universitaire Européen de Florence, Italie bpentimalli@hotmail.com

\begin{abstract}
Awareness and cooperative work in a café-restaurant

In this paper we present a workplace study of the collaborative activity between cooks and waiters in a restaurant/coffee-house. We show how awareness is linked to the attention mechanisms of the participants and how their level of awareness is constantly varying. First of all, using video footage, we analyse the functions of awareness between the co-team members through their peripheral attention, over-hearing and kinaesthetic perceptions : how they need to use shortcuts in their displacements and rapid communication so as to gain time, how they need to avoid collisions in a narrow space, etc. Secondly, based on 'explicitation' interviews conducted with two waitresses, we then present a detailed analysis of the variations in their attention which are linked not only to the cognitive overload present in their current activity but also to the objects and persons which are pertinent for this same activity.
\end{abstract}

\section{KEYWORDS:}

Levels of awareness, foci of attention, cooperative activity, socio-cognitive dynamics, communication, pluri-sensoriality.

\section{1.- Introduction : les niveaux de conscience périphérique (awareness) des activités principales et secondaires}

«Ce que l'on remarque, au sens spécifique du mot, se trouve pris sous un faisceau plus ou moins clair de lumière ; il peut aussi reculer dans la pénombre et dans la pleine obscurité » (Husserl, 1950, p. 319).

La plupart des études sur le travail coopératif sont centrées sur la partie "claire » de l'activité, et analysent les mécanismes observables et explicites de la coordination, ceux qui peuvent être perçus et modélisés relativement aisément.

En vue de clarifier la dynamique de la coordination et des communications dans les lieux de travail, et d'essayer de fournir des indications utiles pour la conception artefactuelle et organisationnelle des lieux de travail, nous développerons ici dans quelle mesure l' «awareness » (que l'on traduira malaisément en français par «conscience périphérique ») de l'activité des collègues peut être cruciale pour le travail coopératif et la coordination, même si elle est mise en place à la marge de l'activité principale. Le travail sur la conscience périphérique nous permet de prendre en compte la partie «obscure » de l'activité, plus implicite et parfois non directement observable, mais qui constitue une partie importante des pratiques effectives du travail coopératif.

Notre objectif est de clarifier la notion de conscience (awareness) de sorte qu'elle éclaire les observations et autres données obtenues dans une situation de travail coopératif, la collaboration de ser- 
veurs et de cuisiniers dans une brasserie. Notre approche ne vise pas des recommandations de conception pour des systèmes spécifiques, mais tente de décrire dans le détail la façon dont la collaboration sociale se construit dans des lieux de travail afin d'esquisser des principes généraux pour la conception.

Dans le domaine du CSCW ${ }^{1}$, on trouve des références à la notion d' " awareness » avec la définition suivante : le fait de rendre l'activité des autres explicite et visible dans des collecticiels de sorte à savoir ce que les autres sont en train de faire (e.g. Mark, Fuchs, \& Sohlenkamp,1997 ; Sandor, Bogdan, \& Bowers, 1997 ; Stiemerling, \& Wulf, 1998). Nous considérons ici que la conscience périphérique n'inclut pas seulement les activités explicites et visibles, mais également les compétences implicites et subtiles des acteurs qui font attention à ce qui se passe autour d'eux via différentes modalités sensorielles (audition, vision, kinesthésie, ...). Ce en quoi cette attention périphérique peut être cruciale dans des situations coopératives va être développé dans le cadre d'une étude de cas.

Nous différencierons donc, comme O'Day, Bobrow, Bobrow, Shirley, Hughes et Walters (1998), la notion d' " awareness » (étroitement liée aux processus attentionnels), et la notion d'audience. Ces auteurs étudient la conception dans le changement de pratique avec passage d'un environnement physique à virtuel, et ont identifié différentes dimensions importantes pour la conception d'environnement d'apprentissage en réseau, dont la dimension de l' "audience », qui correspond aux choix pour établir des actions publiques ou privées, et décider qui peut voir quoi et quels sont les différents niveaux et styles de participation. Ils différencient la notion d' "attention/awareness » et la relient au fait que des participants engagés dans plusieurs activités en parallèle peuvent rencontrer des difficultés attentionnelles.

Le modèle spatial de la conscience périphérique (Benford, \& Fahlen, 1993) souligne aussi le rôle de l'attention dans ce processus. Selon ce modèle, le niveau d' « awareness » que l'objet A a de l'objet B dans le media M (audio, visuel,...) est fonction du focus (ou attention) de A dans $M$ et du nimbus (ou présence) de B dans M. Plus un objet est dans votre nimbus, plus il est conscient de vous, et plus un objet est dans votre focus, plus vous êtes conscient de lui ; l' "awareness » dépend de la façon dont je dirige mon attention sur vous (focus) et de la façon dont vous manifestez votre présence ou activité sur moi (nimbus). A partir de ce modèle, certains auteurs quantifient les niveaux d' «awareness » (e.g. Rodden, 1996). Notre étude, qui décrit comment les acteurs sociaux dirigent leur attention en situation réelle de travail, apporte un support de développement pour ce type de modèles formels qui nécessitent d'être raffinés quant aux mécanismes psychologiques impliqués.

Un nombre croissant d'études de situations de travail analysent la collaboration émergeant entre les participants co-présents ou distants qui sont souvent engagés dans des activités distinctes mais interdépendantes. Par exemple, les études de terrain de type ethnométhodologique du groupe de recherche «work, interaction and technology» du King's college (Heath, \& Luff, 1996; Heath, \& Hindmarsh, 2000) qui portent sur des environnements de travail complexe (centres de contrôle pour les transports urbains, salles de rédaction, ...) explorent comment les gens coordonnent systématiquement leurs activités avec les contributions des autres, même s'ils peuvent être engagés dans des activités distinctes et apparemment sans rapport. Ils soulignent comment les participants au travail collaboratif restent sensibles aux activités co-occurrentes des autres et aux changements dans l'environnement local. Ces auteurs considèrent l' "awareness » comme un flux continuel, émergent au fur et à mesure, pendant le cours dynamique des actions et interactions des participants (Heath, \& Luff, 2000).

Nous considérons également que la variation des niveaux de conscience périphérique est un point important. La conscience périphérique dépend de mécanismes attentionnels qui varient selon l'acti-

1. Computer-Supported Cooperative Work 
vité de l'acteur et selon des caractéristiques des éléments de l'environnement, comme leur saillance et leur pertinence pour l'acteur impliqué dans la situation de travail. Nous allons développer ci-dessous certains concepts qui sont liés à cette variation de conscience.

\section{1.- Niveaux de conscience périphérique et concepts liés}

Dans la littérature en sciences humaines et sociales, on trouve différents concepts qui, même s'ils sont issus de cadres théoriques très différents, permettent de comprendre ce qu'est la conscience périphérique :

- la notion développée par Goffman, sociologue des interactions, de canaux d'activité secondaire qui sont activés à la marge de l'activité principale ;

- l'analyse husserlienne, en philosophie phénoménologique, des variations d'attention, d'active à passive, et des foyers simultanés ;

- L'idée en psychologie cognitive de ce qui est potentiellement accessible par les sujets, que l'on trouve dans la notion piagétienne d'activité pré-réflective, et dans la notion de manifesteté de Sperber et Wilson (1986).

Ces auteurs de différentes disciplines ont développé ces notions qui nous permettent de définir l' «awareness » plus précisément et d'en considérer l'aspect dynamique.

\subsection{1.- De l'activité principale aux activités secondaires}

Goffman (1974) a développé la notion de canaux d'activité secondaire pour décrire ces activités qui sont davantage dans l'ombre mais interviennent en parallèle de l'activité principale; il décrit ces activités secondaires comme étant organisées en quatre canaux latéraux : les canaux de superposition, dissimulation, direction et distraction, qui sont activés en marge de l'activité principale. Ces canaux sont subordonnés à l'activité principale et permettent de traiter des actes ou des événements de manière dissociée. Par exemple, le canal de superposition donne la possibilité de recevoir des messages oraux ou écrits, sans être rendu trop inattentif à l'action principale ; cela montre que l'attention peut être partagée et que l'on peut traiter différents événements en parallèle. La plupart du temps on trouve un foyer d'attention officiel et d'autres lignes d'activité qui sont « hors cadre ». Goffman considère que l'activité officielle (dans le sens où c'est l'activité socialement montrée) est l'activité principale ; nous pensons plutôt que l'activité principale est à définir comme l'activité qui occupe le centre de l'attention du sujet et qui l'intéresse (le capte) le plus, même s'il ne s'agit pas de l'activité officielle. Je peux par exemple parler à un collègue et en même temps écouter vaguement le bruit de l'imprimante pour savoir si mon impression est terminée ; je peux lire un article et en même temps voir vaguement qui entre dans la pièce et identifier si c'est la personne que j'attends ; je peux parler à mes collègues et être en même temps légèrement sensible aux mouvements de la personne assise à côté de moi ; dans ces exemples, l'activité principale est aussi officielle, mais il n'en est pas ainsi dans la situation suivante : je conduis et, pendant trente secondes, je suis totalement absorbée par mes pensées concernant un événement qui s'est produit la veille, et je ne fais pas très attention à ma conduite (juste une attention légère) alors que le souvenir occupe tout l'espace de mes pensées ; après coup je me demande comment j'ai pu conduire pendant ces trente secondes où j'étais perdu dans mes pensées et où je conduisais sans y penser nullement, de façon automatique. Dans cet exemple, l'activité officielle (conduire) n'est pas la principale, dans le sens qu'elle n'est pas l'activité qui est le principal centre de mon attention mais seulement celle que j'ai délibérément choisie comme étant l'activité prioritaire. 


\subsection{2.- De l'activité focalisée à l'activité non-focalisée}

Husserl $(1970, \S 17)$ a décrit les variations du centre de l'attention avec des métaphores spatiales : selon cet auteur, il y a une pluralité de données qui ont simultanément un impact sur le sujet; ces données sont plus ou moins insistantes et ont une force d'impression sur le sujet; quand un objet ressort d'un fond homogène, il insiste, attire le sujet et celui-ci cède à la stimulation, il s'oriente vers lui, l'objet passe alors de l'arrière-plan à l'avant et devient plus proche du sujet ${ }^{2}$. Le sujet peut accepter la stimulation à différents degrés d'intensité ; l'orientation vers l'objet peut être secondaire, une très courte abduction sans attention détaillée. Il y a donc un mode passif de conscience de l'environnement qui est différent du mode actif de saisie des objets. Le point principal de la description de Husserl est que plusieurs foyers attentionnels peuvent co-exister et que le sujet peut avoir des niveaux de conscience variés, d'active à passive. Dans l'exemple de la conduite, je peux, pendant un instant, être centré sur mes pensées, mais maintenir néanmoins une attention légère sur la route et les autres véhicules environnant. Quand je lis un journal, je suis focalisée sur la compréhension de ce que je lis, mais je suis néanmoins conscient de la lumière, et si cette lumière change soudainement je le remarquerai, ou si on me demande ensuite s'il faisait sombre ou clair dans la pièce, je pourrai généralement répondre.

\subsection{3.- De l'activité réfléchie à l'activité pré-réfléchie}

La potentialité nous semble également centrale pour la notion d' «awareness », et un élément qui est potentiellement accessible ou perceptible (comme le son d'une voiture qui passe dans la rue auquel je n'aurai prêté aucune attention mais qui fait néanmoins partie de mon champ perceptif) renvoie à ce qui peut rester pré-réfléchi pendant l'activité. Un objet ou une activité est pré-réfléchie quand il n'a pas été intégré d'une façon conceptuelle ou représentée (sous quelque forme que ce soit), quand il n'a pas été « réfléchi », rendu accessible à la conscience réfléchie. Piaget, en étudiant comment les enfants deviennent conscients de leurs actions (Piaget, 1974), a observé que l'action peut rester au niveau sensoriel et moteur et ignorée par l'enfant, qui peut réussir ses actions sans savoir comment il les réalise. Il en devient conscient lorsqu'une régulation active est nécessaire, quand le comportement automatique n'est plus adapté. Un haut niveau de conscience est donc obtenu par paliers progressifs d'intégration conceptuelle de la conduite automatique et ces observations sur la conscience des enfants peuvent être transférées aux situations de travail coopératif : les activités pour la coordination du travail peuvent avoir ces différents degrés de réfléchissement, des conduites les moins réfléchies aux conduites les plus conscientes. Si l'on vous demande de décrire précisément comment vous lacez vos chaussures, vous aurez probablement besoin d'un temps pour « réfléchir » cette activité qui est automatique et pré-réfléchie (idem si l'on vous demande si une voiture est passée dans la rue). Vermersch $(1994 ; 1999)$ développe dans ce sens une psycho-phénoménologie de l'action.

La notion de conscience périphérique ouvre le champ de la recherche en incluant non seulement ce que l'on fait ou perçoit en sachant qu'on le fait ou qu'on le perçoit, mais aussi les actions et perceptions pré-réfléchies qui sont potentiellement accessibles, potentiellement réfléchies. La notion de «manifesteté » développée par Sperber et Wilson (1986) nous semble également rejoindre l'idée de considérer ce qui se passe au-delà des connaissances explicites. Un exemple de ces auteurs indique clairement cette ouverture : si une voiture passe sous ses fenêtres de façon audible, John peut ne pas y prêter attention et ainsi il ne sait pas, dans le sens le plus faible de « savoir », qu'une voiture passe par là. Sperber et Wilson proposent la notion de «manifesteté » et posent que, sans être connu ou

2. Ces métaphores spatiales de Husserl ne signifient pas que le sujet est toujours passif dans l'orientation de son attention et dépendant de la saillance de l'objet. 
cru, sans être représenté mentalement, ce fait est manifeste pour John. Selon ces auteurs, un fait est manifeste pour un sujet s'il peut être potentiellement perçu ou inféré par lui.

On pourrait dire en d'autres termes que John a un faible niveau de conscience de ce fait; il ne le connaît pas mais il en est potentiellement conscient. Leur notion de manifesteté est plus large que les notions de connaissance ou de croyance car elle inclut non seulement les faits de l'environnement dont le sujet a quelque connaissance, mais aussi ceux dont il peut avoir connaissance. Il y a différents niveaux de manifesteté selon l'accessibilité du fait qui est manifeste.

Ce type d'approche en termes de degrés nous semble fructueuse, contrairement à des positions comme celles de Endsley (1995) qui limitent l' " awareness » de la situation à la connaissance qu'a l'opérateur de l'état de l'environnement dynamique, y compris les états passés et futurs, tel un schéma décrivant explicitement l'état de la situation. L' " awareness » renvoie aussi aux objets qui sont plus ou moins saillants, plus ou moins accessibles mentalement ou perceptivement par le sujet, et il y a des degrés de conscience périphérique comme il y a des degrés de manifesteté.

\subsection{4.- Niveaux de conscience périphérique (awareness)}

On peut distinguer des niveaux de conscience périphérique (awareness) qui sont liés aux notions développées ci-dessus, et considérer que la conscience qu'a John de la voiture qui passe sous ses fenêtres dépend de la répartition de son attention sur les activités principales et secondaires, de la pertinence de ce phénomène pour lui et de sa saillance (plus ou moins bruyant par exemple). On verra en quoi ces perceptions, réalisées à la marge de l'activité principale et qui ne sont pas directement accessibles par le sujet, sont importantes pour comprendre comment les processus de coordination fonctionnent.

Pour synthétiser les notions vues ci-dessus, on dira que le niveau de conscience est limité pour les activités secondaires avec une attention non-focalisée, et que leur vécu est pré-réfléchi et reste dans un mode implicite. Le niveau de conscience est plus élevé pour les activités principales avec attention focalisée et leur vécu est davantage réfléchi.

\section{2.- Etude de terrain : conscience périphérique (awareness) et coopération dans un café-restaurant}

Nous allons maintenant décrire, données empiriques à l'appui, les niveaux changeants de la conscience périphérique, les différentes modalités de l'attention entre serveurs et cuisiniers qui travaillent dans un café ainsi que les fonctions opérationelles de la conscience des actions des collègues.

\section{1.- L'organisation de l'espace de travail}

L'étude de terrain — au cours de laquelle nous allons essayer de «déméler» les processus d'attention périphérique [dans le sens de Heath, Jirotka, Luff et Hindmarsh (1995) "unpack collaboration" pour décrire les modalités subtiles et opaques de la coordination] — porte sur les pratiques de coopération accomplies entre collègues dans un café-restaurant (où les clients viennent pour consommer des boissons ou manger des sandwiches, tartes, salades et plats chauds). Dans l'espace de travail partagé - en particulier l'espace le long du comptoir du bar - tout mouvement, échange langagier et geste des autres ainsi que l'emplacement et l'utilisation des artefacts communs sont mutuellement accessibles aux coéquipiers en co-présence.

Il s'agit d'une situation de travail soumise à des contraintes temporelles et spatiales dans la mesure où sept serveurs et deux cuisiniers - qui coopèrent à satisfaire la clientèle du bar — doivent pro- 
duire rapidement un objet culinaire (la commande du client) dans un espace très étroit situé derrière le comptoir du bar où sont localisés :

- les différents artefacts communs (la caisse, la machine à café, le four, le four à micro-ondes, ...) ;

- l'entrée de la petite cuisine où un premier cuisinier (C1 sur le plan suivant), qui est aussi le patron, prépare les sandwiches et les salades ;

- le micro qui permet de communiquer avec les cuisines du bas (en sous-sol) où un deuxième cuisinier (C2) prépare les plats chauds.

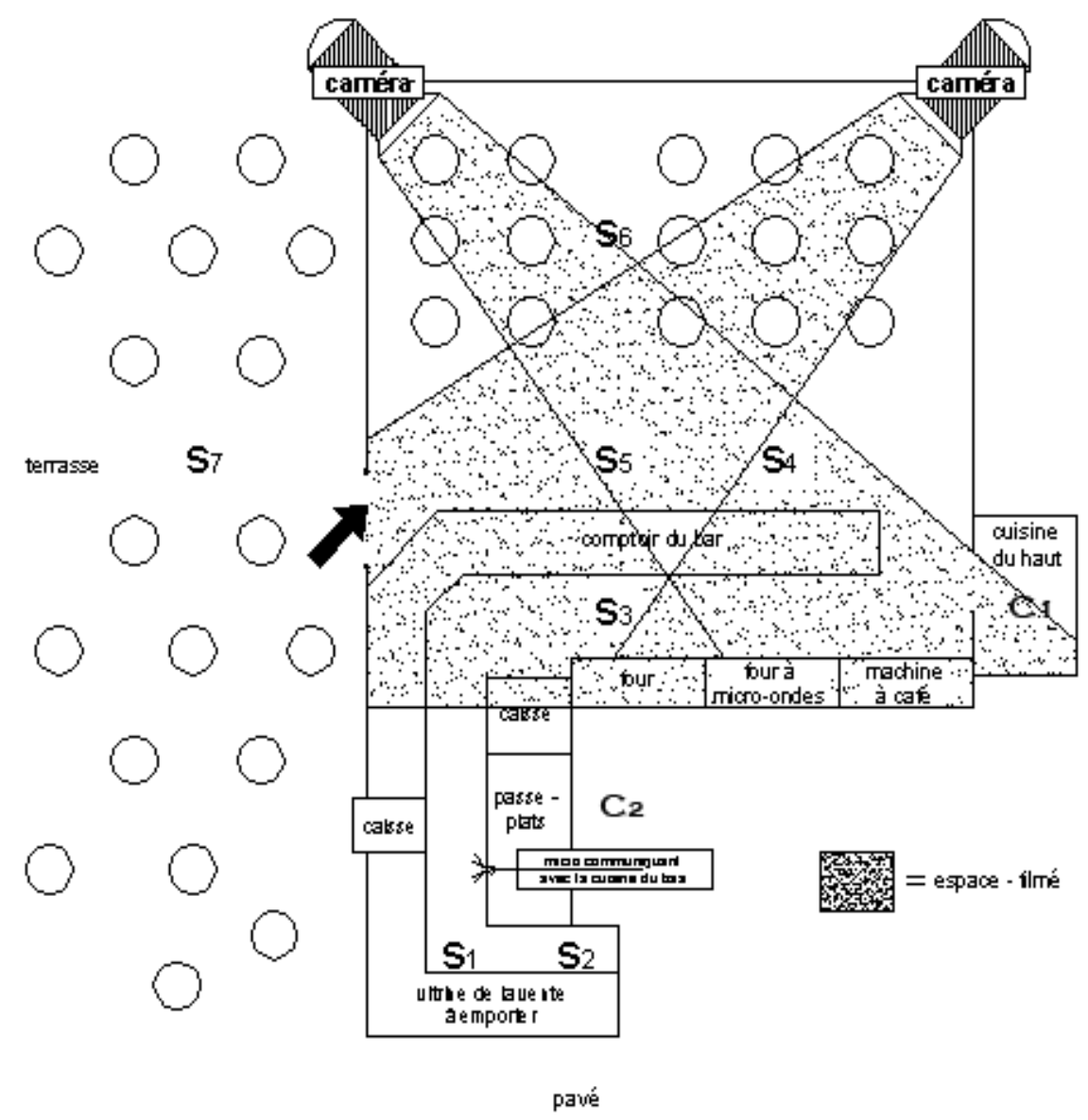

Figure 1.- Plan du café avec les serveurs ( $\mathrm{Sx})$ et les cuisiniers $(\mathrm{Cx})$

Dans cet espace de travail, les activités de coordination se réalisent principalement :

- entre serveurs et cuisiniers pour parvenir à préparer plats et sandwiches ;

- entre les différents serveurs qui se prêtent mutuellement assistance lorsque l'un d'eux est surchargé, et qui se coordonnent afin d'éviter des déplacements inutiles ;

- entre le premier cuisinier (C1) (qui a sa petite cuisine au rez-de-chaussée au niveau du café) et le deuxième cuisinier (C2) (dont la cuisine est située au sous-sol) par l'intermédaire des serveurs qui passent souvent devant le micro communiquant avec la cuisine du bas, et crient les commandes ( $\mathrm{C} 1$ a besoin de frites préparées par $\mathrm{C} 2$ par exemple).

Il s'agit là d'un contexte de travail qui peut paraître simple à première vue alors qu'il s'avère très complexe lorsqu'on observe tous les «parcours d'action » menés par les serveurs et les cuisiniers qui doivent accomplir simultanément : 
- une tâche individuelle (préparer et amener les commandes des clients) vers laquelle en général ils orientent une attention soutenue ;

- une tâche collective de coordination qui mobilise une attention peu focalisée, périphérique (awareness) pour avoir conscience de ce qui se passe dans l'environnement immédiat de travail : les actions des collègues, les artefacts en cours d'utilisation et l'état des commandes, ... Olga (S3 sur le plan) est une exception dans la mesure où, même si officiellement elle est chargée de la vaisselle (remplir et vider le lave-vaisselle, laver et essuyer les verres, ...), son activité réelle consiste principalement à aider les autres; c'est pour cela qu'elle maintient de façon continue une attention et souvent une « conscience élevée » des activités des collègues.

De plus, à l'heure du repas, lorsque le café est rempli par les nombreux clients, les serveurs travaillent sous contrainte de temps et parcourent souvent l'espace en courant pour accomplir leurs tâches.

\section{2.- Méthodologie}

Notre étude s'intéresse aux dynamiques socio-cognitives et aux pratiques de coopération entre serveurs et cuisiniers dans un café, en les analysant à partir $d u$ point de vue de l'observateur mais également à partir du point de vue des participants pour savoir comment elles sont perçues et vécues par les acteurs eux-mêmes. Le point de vue des participants est important dans la mesure où il complète l'analyse du comportement observable (comportement qui est parfois difficile, voire impossible à comprendre du point de vue d'un observateur externe).

Notre étude combine donc deux types d'approches :

- une approche de type ethnométhodologique s'intéressant aux pratiques sociales observables, comme l'abilité à comprendre rapidement, à partir du comportement observable des collègues, le sens des activités dans lesquels ils sont engagés pour voir si les coéquipiers sont une ressource ou une entrâve à l'accomplissement de leur propre tâche, ... ;

- une approche psycho-phénoménologique s'intéressant aux activités cognitives des acteurs telles qu'ils les ont vécu et peuvent les verbaliser (Vermersch, 1994), comme leur compétence à maintenir simultanément différents foyers d'attention afin de mener leur propre activité dans un contexte social de coopération.

Nous adoptons ces deux types d'approches en les considérant complémentaires malgrès leurs objets d'étude différents (l'ethnométhodologie s'intéressant aux pratiques collectives observables et l'approche psycho-phénoménologique s'intéressant aux processus vécus du point de vue de l'individu).

Nos techniques de recueil de données sont, dans leur ordre chronologique :

- des interviews et observations préliminaires afin de se familiariser avec l'organisation du travail "officielle" (l'assignation des postes, la distribution des tâches, les objets et les artefacts de travail, les régles de coopération...);

- des enregistrement vidéo des activités de travail des serveurs et cuisiniers aux heures de repas pendant trois jours (deux caméras filmaient l'espace autour du comptoir caractérisé par l'intensité des interactions entre collègues) afin de voir comment se réalise en contexte l'activité de coordination. Les actions observables sur l'enregistrement vidéo (par exemple les réactions des serveurs aux annonces criées par le cuisinier) ne révèlent cependant pas les changements d'attention qui se réalisent de façon interne pour les acteurs.

La collecte de données vidéo devient classique en ethnométhodologie dans la mesure où elle permet justement de faire une analyse d'un point de vue externe, celui du chercheur. Pour compléter cette analyse, nous nous sommes également intéressés au point de vue de l'acteur. 
- des entretiens d'autoconfrontation (ou post-verbalisations) menés avec certains acteurs qui étaient invités à regarder et à commenter les enregistrements vidéo lorsque certaines séquences d'actions et de communications semblaient difficiles à interpréter par les deux chercheurs. Les techniques d'auto-confrontation ont connu un large développement et sont actuellement souvent adoptées pour des recherches en psychologie et ergonomie cognitive (Theureau, 1992).

- des entretiens d'explicitation (Vermersch, 1994 ; 1999) pour guider les acteurs vers l'évocation et la description détaillée de moments particuliers de leur activité et plus particulièrement ici pour leur faire remémorer les processus attentionnels et la dynamique de la conscience périphérique. Lorqu'on invite les sujets à parler de leurs activités cognitives, une précaution à prendre est d'éviter de leur demander pourquoi ils ont agi de telle ou telle façon ou pourquoi ils ont exprimé de tels jugements, sans quoi ils auraient tendance à rationaliser et à reconstruire leur activité (Ericsson, \& Simon ; 1984; Klein, 1995). L'intervieweur doit au contraire aider les acteurs à diriger leur attention et description sur ce qu'ils ont fait, perçu et pensé. Les techniques d'explicitation - développées par Vermerch - accompagnent les sujets vers l'évocation d'un moment particulier de leurs activités passées, situé dans un temps et un espace spécifique et non pas vers le récit généralisant d'une situation type ; les interviewés remémorent leur activité d'une façon plus directe, plus vivante et moins conceptuelle. C'est ainsi qu'on parvient à une évocation d'épisodes particuliers plutôt qu'à un raisonnement intellectuel sur l'activité, et les acteurs décrivent avec plus de détails ce qui s'est effectivement passé et ce qu'ils ont effectivement fait. L'intervieweur prend en compte des indices de la conduite de l'interviewé (regard, gestes, prosodie, contenu du discours) pour comprendre s'il est en train de raconter son expérience authentique ou si au contraire il est en train de la commenter d'une façon plus distante. L'intérêt de cette technique est double: d'une part, les données obtenues sur les activités cognitives des acteurs (tels que les différents niveaux d'attention) sont beaucoup plus détaillées et exhaustives ; d'autre part, ces mêmes données sont plus fiables que celles récoltées par une interview de type classique dans la mesure où la personne interviewée est guidée vers une évocation vivante de la situation en évitant les reconstructions rationalisatrices.

L'analyse de nos données empiriques ainsi récoltées vise à identifier:

- les fonctions de la conscience périphérique dans une situation de travail, ainsi que l'intérêt et les limites des processus d'attention;

- les spécificités des modes de coordination et de communication dans une situation de travail caractérisée par des contraintes spatiales et temporelles, et plus particulièrement les différentes modalités permettant d'avoir conscience de l'état actuel des actions des collègues, grâce à une écoute flottante et une vision périphérique (Heath, \& Luff, 1994 ; Joseph, 1994) ;

- les compétences à diriger son attention sur la tâche individuelle et sur ce qui se passe alentour (objets, artefacts et collègues).

L'analyse sera présentée en deux parties :

- Les fonctions de la conscience périphérique, soit de ce qui se passe dans l'environnement immédiat de travail, grâce à l'oeil qui traine, l'écoute flottante et les perceptions kynestésiques des serveurs et cuisiniers : leur besoin de communications rapides et de courts déplacements pour gagner du temps, leur besoin d'éviter les collisions dans leur petit espace de travail. Cette partie repose sur une analyse fine de séquences vidéo.

- Les variations de l'attention analysées dans le détail à partir des données recueillies grâce aux entretiens d'explicitation. Avec l'exemple de la commande de Sophie, apparaît le lien entre la conscience périphérique (awareness) et la charge cognitive, tandis que l'exemple du plat de Séverine montre comment la serveuse oriente de façon sélective son attention sur des objets et personnes selon leur pertinence pour son activité. 


\section{3.- Fonctions et dynamiques de la conscience périphérique (awareness) et multiples focalisations de l'attention pendant le travail coopératif.}

Quelle est l'utilité d'une conscience périphérique pour l'exécution du travail quotidien dans un café ? A quoi servent toutes ces activités périphériques accomplies en marge, en parallèle de la tâche en cours de réalisation, telles que laisser « traîner une oreille » (Joseph, 1994) et «maintenir une vision périphérique » pour la réalisation de la coordination collective ?

Dans les activités du café, la conscience périphérique recouvre principalement trois fonctions; elle est utile pour :

- l'économie collective de déplacements et actions, grâce à une vision périphérique des mouvements des collègues ;

- le besoin de communications rapides et non intrusives en écoutant sur un mode périphérique les messages oraux adressés dans le brouhaha du café ;

- le besoin d'éviter des collisions dans un petit espace partagé grâce à des modalités visuelles et kinesthésiques.

\subsection{1.- Conscience périphérique pour l'économie collective de déplacements et actions}

A cause des contraintes spatiales et temporelles qui pèsent sur le travail accompli dans le café, serveurs et cuisiniers essayent autant que possible d'éviter tout déplacement et action inutiles. Pour s'épargner des mouvements, ils doivent organiser leurs propres activités de façon à réduire leur trajet d'action, mais ils essayent également de limiter les mouvements de tout le collectif de travail en ayant conscience :

- des trajets d'action des collègues qui peuvent leur éviter un déplacement ;

- des actions des collègues qui parfois les aident spontanément en leur évitant ainsi de se déplacer...

- des collègues qui sont déjà en train d'utiliser des artefacts dont ils ont besoin.

\section{A) Conscience (awareness) des parcours d'actions des collègues}

Le travail dans le café implique de nombreux mouvements et déplacements de la part des serveurs qui souvent marchent simultanément d'un pas rapide autour du comptoir du bar afin d'utiliser les artefacts, prendre les plats chauds dans le passe-plats et aller chercher les plats froids près de la cuisine du haut ou dans la vitrine de la vente-à-emporter. Ces mouvements et trajets prennent du temps et peuvent créer de petits embouteillages. Les serveurs essayent de minimiser leurs déplacements et mouvements en profitant des parcours d'action de leurs collègues. Ils sont en effet capables de voir, la plupart du temps en vision périphérique, si l'activité en cours d'un collègue peut leur épargner un mouvement et demandent alors au coéquipier d'accomplir une action à leur place. Les deux scènes suivantes (décrites à partir de données vidéo) montrent justement comment la conscience des trajets d'action des collègues donne l'opportunité d'économiser des déplacements :

Olga est en train de laver la vaisselle derrière le comptoir du bar pendant que la serveuse Annie est en train de s'approcher le long du comptoir, mais de l'autre côté. Lorqu'Annie est en train de contourner la fin du comptoir, près de la machine à café, elle dit quelque chose, puis Olga lance un regard dans sa direction et lui demande: “donne-moi le torchon là". Sa requête verbale est accompagnée d'un coup d'oeil vers la machine à café de façon à indiquer à Annie l'endroit où se trouve le torchon. C'est le seul moyen gestuel à disposition pour indiquer l'emplacement vu que ses mains sont déjà occupées à la tâche du lavage. Annie interrompt momentanément la réalisation de sa propre tâche, fait un tout petit détour, attrappe le torchon et le passe à Olga. 
Une des serveuses chargée de la vente-à-emporter, Estelle, est en train de prendre le plat de frites dans le passe-plat pour l'apporter à la cuisine tandis que Stéphanie, l'autre serveuse assignée à la vente-à-emporter, est justement en train de se diriger vers la cuisine. Estelle tend alors le plat vers Stéphanie en lui disant: "prend-le, prend-le, prend-le"; Stéphanie regarde derrière elle, passe le sandwich qu'elle tenait de la main droite à la main gauche, prend le plat de frites dans sa main libre et l'apporte à la cuisine.

Dans ces deux exemples les serveuses, tout en focalisant leur attention sur leur tâche principale en cours de réalisation, regardent autour d'elles (lancent des coups d'oeil, maintiennent un regard sur l'environnement) et voient si le trajet d'action d'un de leurs collègues peut leur éviter un mouvement ou déplacement. Dans les deux petites scènes présentées ci-dessus les serveuses Annie et Stéphanie ne mettent pas volontairement en forme leur action pour que les autres en aient conscience. C'est différent de ce qui se passe dans les salles de contrôle étudiées par Heath et Luff (2000) où le contrôleur met en forme volontairement sa conduite de sorte à permettre aux co-participants d'entendre et de voir les actions «pertinentes » à leur activités. Dans notre exemple, la conscience (awareness) n'est pas mutuelle mais plutôt unilatérale : Olga a conscience des actions d'Annie tandis qu'Annie n'a pas conscience du fait qu'Olga a conscience d'elle et n'a pas l'intention de rendre son action visible à Olga. Annie ne construit pas de façon intentionnelle ses actions pour rendre possible une conscience mutuelle.

Dans les termes de Benford et Fahlen (1993), l'awareness dépend ici seulement de la façon dont la serveuse A oriente son attention vers une collègue $\mathrm{B}$ (focus) et non pas de la façon dont la collègue $\mathrm{B}$ rend visible sa présence et son activité à la serveuse $\mathrm{A}$ (nimbus).

L'activité des collègues, les trajets d'action ou le besoin d'aide peuvent également être inférés en remarquant l'état des artefacts utiles au travail (l'emplacement, la configuration, ...). Par exemple dans le café, la serveuse peut savoir, à cause d'un plateau où sont posées trois petites assiettes à café, qu'un collègue a besoin de trois cafés. Pour connaître l'activité des collègues, l'acteur établit des déductions à partir de l'état des objets et artefacts de travail (Dix, 1997).

\section{B) Conscience (awareness) des « coups de main » apportés par les collègues}

Il existe également dans le café, une forme d'entraide spontanée pour permettre à un collègue d'économiser un déplacement. Ces coups de main sont habituellement donnés par ceux dont la tâche officielle est d'aider autour d'eux, comme Olga ou Mme V., ou par les autres dans leurs minutes de temps morts ou lorsque leur charge de travail est faible; les serveuses parlent clairement au cours des entretiens de leur disponibilité à aider spontanément les autres, sous réserve qu'elles ne soient pas trop occupées par leurs commandes, auquel cas elles ne voient, n'entendent, et ne devinent pas les besoins des autres.

Des problèmes peuvent surgir quand les serveurs ne s'aperçoivent pas qu'ils ont été aidés; ce sont justement les risques et les difficultés engendrés par ces modes de coordination faibles, implicites et non intrusifs. C'est le cas dans la scène vidéo suivante où la serveuse Mme V (S4 sur le plan), a apporté son aide à une autre serveuse, Stéphanie (S1), en transférant un sandwich qui était adressé à Stéphanie du comptoir vers un emplacement situé dans l'espace de travail de sa collègue. Stéphanie n'a pas vu l'action accomplie par Mme V, n'a pas conscience du transfert et attend son sandwich :

Le cuisinier M.V quitte sa cuisine un sandwich à la main, en disant à Mme V de l'apporter à Stéphanie qui travaille à la vente-à-emporter; Mme $\mathrm{V}$ prend le sandwich, l'apporte de l'autre coté du café et le pose sur le comptoir de la vente-à-emporter sans rien dire. Stéphanie, la destinataire, engagée dans une autre tâche ne s'aperçoit pas du transfert. On voit Stéphanie et Mme V très proches l'une de l'autre, chacune le dos tourné, et Stéphanie ne semble pas avoir conscience des mou- 
vements de ses collègues. Quelques minutes plus tard Stéphanie — située au tournant près de la caisse - attend son sandwich en regardant vers la cuisine; elle se met régulièrement sur la pointe des pieds et regarde ce qui se passe dans la cuisine, puis elle se dirige vers la cuisine en traversant avec difficulté le petit couloir encombré et y rentre. Surgit alors la voix du cuisinier qui d'un ton fâché s'exclame : "je viens tout juste d'en faire deux, là, ma femme te les a donnés!" ; Stéphanie sort de la cuisine avec une expression offensée et, tout en reparcourant le couloir derrière le comptoir, dit : "mais personne me le dit !" pendant que le cuisinier indique la fin du comptoir en disant "regarde, ils étaient là".

Mme V qui voulait essayer d'éviter un déplacement à Stéphanie, a surestimé la conscience (awareness) potentielle de sa collègue qui, en fait, n'a pas perçu ses actions pour l'aider et qui aurait alors préféré un mode de coordination plus explicite où "quelqu'un lui dise...".

Cet exemple montre clairement les avantages et les risques possibles dûs à ces modes non intrusifs de coordination collective ; l'entraide mutuelle économise du temps et des déplacements à condition qu'elle soit perçue par les personnes qui sont aidées.

\section{C) Conscience (awareness) de l'utilisation des artefacts par les collègues}

Dans la mesure où les serveurs partagent des artefacts de travail tels que la caisse, la machine à café, le four et le four à micro-ondes, ils doivent souvent attendre qu'un artefact soit libre. S'ils n'ont pas une vision périphérique et une conscience anticipée de la disponibilité d'un artefact (quelqu'un est-il en train de l'utiliser ?) ils perdent du temps et accomplissent des trajets inutiles. C'est le cas d'Annie dans la scène suivante :

La serveuse, Séverine, est en train d'utiliser la caisse pendant qu'Annie, une autre serveuse, se dirige vers l'artefact en question sans regarder devant elle, les yeux penchés sur son carnet de commande qu'elle tient à la main ; puis, en levant les yeux, elle voit que Séverine utilise l'artefact dont elle a besoin. Annie semble hésiter quelques secondes, elle effectue un petit tour sur elle-même et finit par s'approcher de la caisse pour attendre la fin de l'activité de sa collègue. Quelques secondes plus tard, dès qu'elle entend la voix du cuisinier annonçant son prénom en posant un plat sur le comptoir, elle s'en va prendre le plat en question, et la caisse se libère.

La disponibilité d'un artefact est une condition préalable pour pouvoir accomplir certaines actions qui sont centrales à la tâche ; l'utilisation de la caisse est probablement un objectif conceptualisé, et vérifier sa disponibilité est une activité nécéssaire qui se réalise en parallèle pendant qu'on est en train de faire autre chose. Des petits contrôles visuels (Sudnov, 1972) sont indispensables à une prise de conscience (awareness) de l'activité des collègues afin d'éviter de perdre du temps et se déplacer inutilement.

La serveuse Annie est débutante, il est possible en effet d'observer qu'elle hésite dans ses trajets d'action et ne tire pas profit de chaque mouvement comme le fait Séverine, serveuse expérimentée. Un comportement typique d'une serveuse expérimentée consiste à toujours remplir son plateau et à effectuer des déplacements profitables en évitant tout trajet à vide. Une des difficultés pour une débutante est justement de maintenir simultanément différents foyers d'attention et de maîtriser en parallèle plusieurs activités. Par exemple, pendant qu'elle remplit son plateau, la serveuse peut savoir qu'un artefact est libre pour sa prochaine action, qu'un client est en train d'attendre de faire sa commande, qu'elle doit porter un verre d'eau à un autre client ou que le cuisinier est en train d'annoncer sa commande, ... 


\subsection{2.- Conscience (awareness) des communications rapides et non intrusives}

Une activité sous contrainte de temps — comme celle qui a lieu dans le café à l'heure du déjeuner implique également une spécificité dans les modes de communication qui reposent plutôt sur une « écoute périphérique » que sur des échanges explicites.

Une attention auditive flottante est indispensable dans deux cas au moins ;

- pour le cuisinier qui doit entendre les commandes orales des serveuses ;

- pour les serveurs qui doivent entendre les annonces et les requêtes du cuisinier.

A) Le cuisinier doit toujours maintenir une attention auditive flottante dans la mesure où les deux serveuses chargées de la vente-à-emporter lui transmettent leurs commandes oralement, en criant pour parvenir à couvrir les autres bruits du café ; une distance de 8-10 mètres les sépare (les autres serveurs lui disent en passant devant sa cuisine ou ils lui déposent un billet). Une scène fréquente le montre clairement :

Le cuisinier est en train de préparer des sandwichs dans sa cuisine ; il peut entendre Stéphanie qui, de la vente-à-emporter, crie : "un américain-saucisse!"; sans pour autant interrompre son activité et sans lancer un regard vers la serveuse, il continue tout simplement son activité précédente.

Le cuisinier ne donne aucun signe réactif (feedback) qui indiquerait à la serveuse qu'il a bien entendu sa commande; la réception du message est ainsi non intrusive, non dérangeante pour la tâche principale en cours de réalisation (la préparation du sandwich). La communication nécessaire à la coordination semble être ici une activité secondaire se réalisant en parallèle sans déranger l'activité principale en cours de réalisation. Schmidt avait déjà souligné que l'acquisition de l'information concernant la conscience mutuelle (mutual awareness) n'est pas intrusive dans le sens où elle n'intervient pas dans le cours du travail en imposant qu'une réponse soit exprimée (Schmidt, 2000).

Sperber et Wilson (1986) affirment que la communication est toujours incertaine et risquée vu qu'on ne sait jamais comment l'autre va interpréter le message. Dans la situation du travail étudiée, une activité sous contrainte de temps, les acteurs doivent prendre encore plus de risques et doivent accepter l'incertitude de ce qui a été entendu. Il arrive bien sûr que le cuisinier n'entende pas ou oublie une commande en obligeant ainsi les serveuses à reformuler leur demande après un bout de temps. La conséquence de ces petits dysfonctionnements dans la communication entre collègues est l'insatisfaction du client en attente de sa commande; ce n'est pas un risque dramatique mais - dans un contexte commercial - il s'agit quand même d'une conséquence négative.

B) Les serveurs et les serveuses ont également besoin de maintenir une attention auditive flottante pour s'apercevoir si leur sandwich est prêt; quand c'est le cas, le cuisinier pose l'assiette sur le comptoir du bar et annonce leur prénom suivi du type de sandwich ("Stéphanie, jambon") ou il crie seulement leur prénom.

Nous soulignons encore une fois, au delà des avantages qui permettent de gagner du temps et de maintenir son attention focalisée sur la tâche principale en cours de réalisation, les difficultés et les risques des modalités de ce travail d'articulation. Quand un acteur est engagé dans une activité exigeant beaucoup d'attention et/ou mobilisant une importante charge cognitive, il peut alors ne pas avoir la disponibilité supposée par ses coéquipiers et peut ne pas être capable de maintenir l'attention auditive diffuse qui est requise par ses collègues. La scène suivante le montre bien :

Annie est en train de prendre un plateau où sont posés plusieurs plats, tout en parlant avec Mme $\mathrm{V}$ et en se dirigeant vers des clients assis à une table de la salle; pendant ce temps là, le cuisinier sort de sa cuisine et appelle : "Annie, Annie" tout en posant un plat sur le comptoir et en regardant vers Annie ; puis une autre serveuse, Séverine, qui passait là, demande "la pizza c'est pour Annie ?", elle prend la pizza, la fait chauffer dans le four à la place d'Annie, la pose à nouveau sur l'assiette et la donne à Mme V en lui disant "c'est pour Annie". De son côté, Annie qui n'a rien entendu ne 
semble nullement avoir conscience de tous ces relais accomplis pour elle.

Cet exemple montre que la conscience périphérique (awareness) ne peut pas être constante et régulière, et qu'il est difficile de « compter sur elle ». Lorsque les acteurs sont engagés dans une activité qui exige beaucoup d'attention ou un effort cognitif important, ils peuvent alors ne pas être disponibles pour recevoir les messages des collègues qui parviennent en parallèle de l'activité principale dans laquelle ils sont engagés. Des études en psychologie cognitive ont montré que le sujet peut accomplir deux activités exigeant une attention sur un mode parallèle si le niveau d'exigence des deux tâches n'excède pas le seuil d'habileté du sujet, qui dépend de la charge mentale induite par les deux tâches (Richard, 1980).

C'est également difficile quand l'activité en cours de réalisation mobilise le même mode sensoriel que l'information qui parvient en parallèle : il est ardu de parler et en même temps d'écouter autre chose (comme Annie dans l'exemple précédent); il est en revanche plus facile de parler tout en regardant quelque chose.

Les serveurs ont également besoin d'entendre si le cuisinier (C1) est en train de demander les frites qui sont préparées dans les cuisines du bas, puisque tout serveur qui «passe par là » a alors le devoir de répondre au cuisinier. Pour ce type de tâches qui ne sont pas assignées à quelqu'un en particulier (tout serveur qui passe près du micro peut commander les frites aux cuisines du bas), la coordination repose sur ce type de conscience périphérique (awareness). Les données montrent que cela marche seulement si la serveuse qui est au bon endroit n'est pas surchargée (sinon elle ne peut pas entendre sur un mode périphérique) ou si c'est dans son intérêt que les frites soient commandées ou soient apportées du passe-plats au cuisinier (comme Sophie qui nous dit avoir répondu au cuisinier parce qu'elle avait besoin des frites «pour éliminer une commande dans ma tête »). Au cours d'un entretien, Séverine décrit le moment où - étant en train de finir la préparation de son plateau - elle a soudain entendu la voix du cuisinier demandant les frites; elle avait tout juste fini de récapituler les actions à peine effectuées (la caraffe, les verres, le pain, les couverts, ...) et commençait à se diriger vers la caisse située près du passe plats. Pendant ce petit laps de temps Séverine peut entendre la voix du cuisinier "parce qu'à ce moment là j'avais fini de préparer mon plateau et j'étais en train d'aller faire mon ticket de caisse ; entre le moment où j' ai fini de remplir le plateau et fais mon ticket de caisse, je n'ai rien à faire" dit-elle.

Les activités accomplies sous contrainte de temps engendrent des formes de communication particulières dans le sens où elles sont restreintes à un minimum d'échanges et où elles sont moins fiables que les communications ordinaires. Comparé aux conversations ordinaires, les acteurs semblent compter beaucoup sur l'attention auditive diffuse de leurs collègues sans s'attendre toujours à un "signal positif de compréhension" (Clark, \& Brennan 1991 ; Clark 1996) pour s'assurer que les collègues les ont bien entendus (les habituels régulateurs comme « oui », «hmm», « okay »). Ils ont tendance à compter sur l'attention flottante de leurs collègues.

La conception d'un nouvel espace et de nouveaux modes de communication pour ce type de situation caractérisée par un important travail collectif, peut s'avérer assez complexe. Il y aurait par exemple la possibilité d'installer un micro entre la cuisine du haut et la vente-à-emporter. Cela pourrait limiter le bruit provoqué par le annonces criées d'un bout à l'autre du comptoir et pourrait engendrer de façon systématique des messages audibles entre le cuisinier et les deux serveuses qui travaillent loin de lui. Mais ce très simple changement technologique pourrait également limiter les pratiques d'entraide mutuelle entre serveurs qui passent près de la cuisine et apportent parfois la commande qui est prête pour ceux de la vente-à-emporter. Les communications par le biais du micro rendraient probablement inaudible aux autres serveurs le destinataire de la commande qui ne pourra ainsi plus être aidé. On devrait alors évaluer si le gain en terme d'une communication plus claire et 
précise entre le service de la vente-à-emporter et le cuisinier serait plus important qu'une perte de conscience périphérique (awareness) de la part des autres serveurs.

\subsection{3.- Conscience périphérique (awareness) pour éviter les collisions dans un petit espace partagé}

Etant donné l'étroitesse de l'espace où serveurs et cuisinier se croisent et utilisent les artefacts de travail, la synchronisation de leurs mouvements et déplacements est cruciale, sans quoi ils seraient toujours en train de se bousculer. Les acteurs semblent avoir développé une perception kinesthésique automatique qui s'ajouterait à la vision périphérique en leur permettant d'éviter des collisions et d'avoir un cetain niveau de conscience de la présence et des mouvements des autres. La scène vidéo suivante implique trois acteurs qui sont très proches les uns des autres et qui ont quelques difficultés à poursuivre leurs parcours d'action à cause des coéquipiers qui créent un petit embouteillage :

Le cuisinier quitte la cuisine un sandwich à la main, tandis qu'Olga est occupée à la machine à café, et qu'Annie s'approche de la zone située prés de la machine à café. Lorsque le cuisinier est en train de donner un sandwich à une autre serveuse qui est juste devant lui, Olga est en train de poser une sous-tasse à café sur le comptoir. Annie parvient à passer en se faufilant entre Olga et le cuisinier, puis lorsqu'elle se trouve près de la cuisine, se fraye un chemin en se faufilant derrière le cuisinier qui est en train de faire demi-tour pour retourner dans sa cuisine pendant qu'Olga tourne sur ellemême.

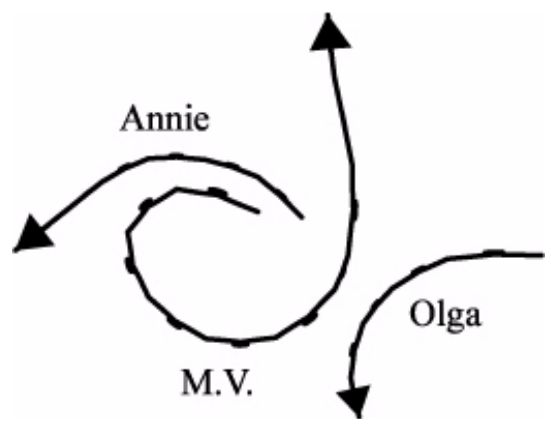

Figure 2.- Schéma de l'engrenage

Les mouvements dans l'espace sont souvent difficiles à décrire, mais on est ici frappé par la synchronisation des trois acteurs : quand le cuisinier tourne sur lui-même il semble que ce mouvement fasse tourner une autre personne comme un engrenage, même s'ils n'ont pas de contact physique entre eux. Les trois acteurs tournent également de façon symétrique. C'est comme s'ils avaient une « conscience corporelle » (bodily awareness) des mouvements des autres grâce à laquelle il seraient capables de synchroniser leurs mouvements et d'éviter des collisions. Cette conscience n'est pas seulement possible grâce à la vision périphérique puisque les acteurs sont parfois dos à dos et ne se voient pas ; elle semble plutôt liée à une perception kinesthésique des mouvements des collègues.

De nombreuses séquences vidéo montrent la formation de petits embouteillages dans l'étroit couloir derrière le comptoir, au cours desquels plusieurs collègues se croisent les uns les autres, chacun engagé dans son trajet d'action particulier tout en ayant cette conscience (awareness) - secondaire mais nécessaire - des mouvements des autres. Lorsqu'on regarde les séquences vidéo au ralenti, cette synchronisation corporelle semble très complexe et subtile. Par moments on a presque l'impression qu'il s'agit d'un ballet pré-organisé, comme les synchronisations des piétons décrites par Goffman (1973), et non pas d'une coordination effectuée sur le moment de façon ad hoc.

Ces modes particuliers de coordination nous amènent sur le terrain des actions pré-réfléchies, dans le sens où les acteurs ont des difficultés à les remémorer et à les décrire. Il s'agit de mécanismes qui 
sont incarnés, inscrits dans l'action et difficiles à conceptualiser mais qui sont néanmoins efficaces et utiles pour que les acteurs évitent des collisions dans un espace étroit. A cet égard, même s'ils correspondent à des niveaux de faible conscience (awareness), ils peuvent également être considérés comme faisant partie des activités secondaires qui ont lieu « hors cadre » par rapport à la centralité de la tâche principale mais qui sont indispensables à la réalisation efficace du travail collectif.

Lorsque le travail coopératif à distance est médiatisé par une technologie, on peut se demander si cette sensibilité corporelle des coprésents ne manquerait pas aux usagers. Il s'agit en effet d'une source d'information sur les collègues qui est souvent ignorée puisque les acteurs ne parviennent pas facilement à avoir conscience des perceptions kinesthésiques. Ces perceptions sont mobilisées de façon automatique afin d'adapter son propre comportement au travail collectif et être sensible à ce qui se passe alentour, mais leur rôle et leurs effets demeurent inexpliquées par les recherches sur la coopération.

Cette analyse des modalités de mobilisation de la conscience périphérique afin de réaliser une action collective avec les collègues qui partagent un même espace de travail, montre que l'écoute flottante, la vision périphérique ainsi que la sensibilité kinesthésique des acteurs leur permet de gagner du temps, d'éviter des déplacements et de créer un système d'entraide mutuelle en maintenant simultanément différents foyers d'attention et différents niveaux de conscience de ce qui se passe autour d'eux.

\section{4.- Analyse détaillée des variations de l'attention}

Nous allons maintenant développer une analyse plus détaillée des variations de l'attention qui permettent à l'acteur d'avoir une plus ou moins grande conscience des événements environnants. Deux exemples vont illustrer ce phénomène d'une conscience périphérique rapidement changeante. Pour étudier de manière plus approfondie les changements rapides de l'attention des sujets, nous avons besoin d'un type différent de méthodologie. Nous avons ainsi utilisé les techniques de l'entretien d'explicitation qui vise à aider le sujet à remémorer et à décrire son activité. Cette technique - qui est difficile à acquérir et exige une formation et un entraînement particulier de la part de l'intervieweur - est particulièrement conçue pour éviter des reconstructions de la part de la personne interviewée (cf § sur la méthodologie).

Pour augmenter les chances de recueillir un souvenir vivace de ces phénomènes, nous avons interwiewé les serveuses tout de suite après leur activité pour faciliter l'évocation d'événements peu éloignés dans le temps.

\subsubsection{Variations de l'attention et charge cognitive}

Nous allons d'abord présenter l'extrait d'un entretien d'explicitation qui fait ressortir les changements rapides de l'attention des serveuses, ainsi que le lien entre les exigences cognitives requises par la tâche en cours de réalisation (ou la charge cognitive de travail) et les compétences des acteurs à avoir une conscience périphérique des activités des collègues. Il existe une interdépendance entre l'activité individuelle et la possibilité de demeurer attentif à la situation collective, comme l'indique très clairement l'exemple suivant, qui montre également les variations qualitatives de l'attention et les changements rapides dans les focalisations de l'attention qui se réalisent pendant l'activité de la serveuse.

L'entretien d'explicitation -dont nous présentons un extrait- a été conduit avec une serveuse du café afin d'améliorer les connaissances sur les changements de focalisations de l'attention dans la réalisation des tâches quotidiennes et sur les différents niveaux de conscience (awareness) indispensables à l'accomplissement du travail coopératif dans le café. Le contexte de l'action décrite est le suivant : la serveuse Sophie, penchée dans la vitrine de la vente-à-emporter, est en train de prendre 
deux gâteaux tout en attendant qu'une autre commande soit prête (elle doit être annoncée oralement par le cuisinier), quand soudain elle entend la voix du cuisinier annonçant que sa commande est sur le comptoir du bar.

I - "là par exemple tu allais prendre les gâteaux, est-ce qu'avant que M.V dise ton prénom tu entends ce qu'il dit ?" (débit ralenti de la voix de l'intervieweur qui a mené l'entretien pendant un moment; la serveuse a désormais retrouvé une mémoire vivace de la situation vécue pendant les heures précédentes ; c'est pourquoi l'intervieweur utilise le temps présent "tu entends ...")

$\mathrm{S}$ - j'entends pas du tout, je suis concentrée dans ce que je fais, y a que quand on m'appelle que j'entends

I - donc tu entends, euh tu entends la voix de M.V au moment où il dit ton prénom ?

S - voilà, autrement si, j'entends du brouhaha mais je fais pas attention, je pense surtout à ma commande, à ce que je fais

I - et quand tu penses à ta commande, tu fais quoi ?

S - je pense à mes gâteaux et je dis "bon, j' ai pris ça, j' ai pris ça, j' ai encore ça à faire, j'ai (rires) j'ai pas fait mon ticket de caisse, j'ai pas..."

I - et quand tu entends des choses comme ça et après, tu vois, juste au moment où tu entends ton prénom, qu'est-ce qui se passe là ?"

$\mathrm{S}$ - il y a un déclic qui se déclenche dans ma tête qui est l'autre commande à passer en premier, j'ai posé mes gâteaux et puis c'est euh l'assiette qui part en premier

I - et puis qu'est-ce qui se passe, tu vois, juste au moment où t'entends ton prénom?

$\mathrm{S}$ - j'entends mon prénom, mon premier réflexe, c'est euh tourner ma tête (elle fait le geste) et après je sais que c'est pour moi, donc je reprends mes gâteaux et hop!

I - est-ce que tu entends le cuisinier dire un autre prénom (...) et tu l'entends de la même façon ou $\ldots$ ?

S - non, c'est pas pareil, "tiens Séverine" c'est, non ça me ferait moins de, j'ai pas le déclic comme j'ai quand c'est mon prénom (...) j'entends mieux, déjà mon esprit se libère de ça, parce que je suis moins concentrée et j'ai entendu mon prénom, ça y est ! j'entends ce que les autres, enfin, je comprends pas mais j'entends mieux ce que les autres disent, je suis moins dans

I - ah donc avant d'entendre ton prénom, disons, qu'est-ce qui se passe avant de l'entendre?

$\mathrm{S}$ - je suis dans mon univers, j'entends les autres mais j'entends sans entendre, je suis en train de me parler à moi même, de me dire “j' ai pris ça, j'ai pris ça euh", et puis une fois que j'ai entendu mon prénom, et je me retourne je vais la chercher (la commande) et par exemple si Olga est en train de me dire une bêtise ou un truc pour rigoler, là je vais l'entendre et je vais comprendre, qu'avant je pouvais très bien l'entendre mais pas la comprendre, et puis pas chercher, et partir quoi (...) mon esprit se libère d'une commande, donc j'ai l'impression que c'est moins lourd (léger rire) à porter que ça y est ! je peux écouter les autres trucs quoi (...) je me dis euh, comment je pourrais dire (en baissant la voix) ... je me dis : ouf ! ça y est, elle est prête, j'ai plus ça à penser, je peux penser à autre chose quoi (...) ou je peux, ça y est ! j'ai une place en plus dans ma mémoire (rire amorcé) et je peux aller rechercher une commande, et donc j'ai entendu mon prénom euh pendant que j'étais en train de prendre les gâteaux, ben là j'étais libérée d'une chose donc je pouvais me consacrer qu'à ma deuxième commande sans être perturbée en me disant : j'ai encore une commande à aller porter ou...

I - et quand tu dis dans ton monde à toi, ça veut dire...?

S - ça veut dire qu'on peut me parler, parler, parce que j'entendrais mon prénom, on peut me dire quelque chose mais je suis censée de me dire, je vais la retrouver la personne, je dis "mais en fait qu'est ce que tu m'as dit, là ?", j'ai entendu sans entendre quoi

I - et si t'es dans ton monde comme ça et le cuisinier parle?

S - voilà c'est la voix du, c'est la seule voix que je peux dire que j'écoute vraiment, que j'entends bien quoi (...) tout d'un coup on entend une voix claire qu'on entend, on entend une voix claire (...) 
voilà après je rentends un peu ce qu'on dit, si ils déconnent, je peux très bien entendre et rigoler quoi (...) donc je pense déjà moins à mes gâteaux, après je reviens dedans".

Le « déclic » ci-dessus décrit, indique le changement dans la focalisation de l'attention, et plus particulièrement le moment où la première commande (celle que la serveuse était en train d'attendre et qui était temporairement en arrière-plan) devient la première occupation de la serveuse même si elle focalise son attention sur sa tâche en cours de réalisation (le choix des gâteaux dans la vitrine). Juste avant d'entendre son prénom, Sophie n'a pas conscience de ce qui se passe autour d'elle, puis quand tout d'un coup elle entend son prénom, elle ressent ce « déclic » qui la détourne de la tâche en cours de réalisation et la pousse à aller servir la première commande tout en ressentant une diminution de sa charge cognitive. La serveuse n'est plus préoccupée par la première commande en attente, et quand son esprit devient « plus léger », elle peut participer à nouveau aux échanges verbaux collectifs entre les coéquipiers.

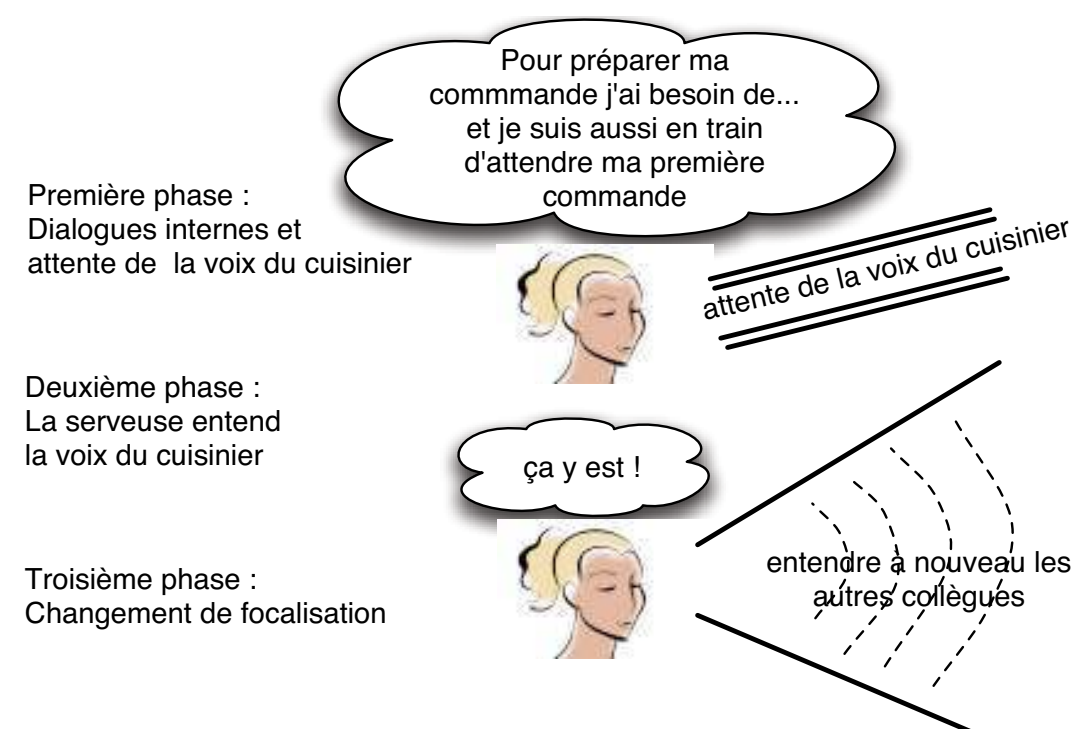

Figure 3.- Schéma du « déclic »

\section{- 1ère phase : Charge cognitive et faible conscience périphérique}

Quand Sophie - qui est engagée dans la préparation de sa deuxième commande — dit "je suis concentrée dans ce que je fais", elle signifie qu'elle est en train de focaliser son attention sur la tâche en cours de réalisation en se parlant à elle-même. L'attente de la première commande occupe aussi son esprit. Cette charge cognitive la prive de la conscience de ce qui se passe autour d'elle.

Trois processus cognitifs sont en oeuvre :

\section{a) Dialogue interne sur l'activité principale}

Lorsque la serveuse prend les gâteaux dans la vitrine elle est train de se parler de façon interne à elle-même afin de récapituler ce qu'elle a fait et de planifier ce qu'elle doit encore faire. Elle est simultanément en train de concevoir des plans « dans sa tête » tout en demeurant sensible aux bruits du café. Sophie est momentanément engagée dans la planification mentale de ses actions mais ensuite sera capable d'improviser et de réagir aux circonstances du moment.

\section{b) Isolement ou faible conscience de l'environnement}

L'activité mentale caractérisée par les dialogues internes conduit à une isolation auditive de ce qui se passe autour, la serveuse n'entend pas vraiment la communication environnante entre les collè- 
gues, à l'exception de l'annonce du cuisinier qui lui est adressée. On peut supposer que les dialogues internes, en plus de l'attente de la voix du cuisinier, surchargent le canal auditif de la serveuse et expliquent les raisons de son incapacité à entendre finement les échanges environnants.

c) Attention non focalisée sur un objet secondaire (l'attente de la commande)

L'attente de la voix du cuisinier l'appelant par son prénom demeure dans l'arrière-plan pendant que Sophie prête attention au choix des gâteaux. L'attente de la première commande — qu'elle soit prête - occupe son esprit.

\section{- 2ème phase : le prénom crié par le cuisinier}

\section{- 3ème phase : changement de focalisation de l'attention}

Le déclic se déclenche tout de suite après que la serveuse ait entendu son prénom: Sophie répond intérieurement ("ça y est !") et ressent une diminution de sa charge cognitive grâce à la suppression de la tension créée par l'attente de la première commande. Le fait qu'elle entende uniquement l'annonce rapide criée par le cuisinier qui émerge du bruit de fond montre qu'elle accomplit un filtrage auditif de l'information. La serveuse est capable de distinguer l'annonce du cuisinier dans le brouhaha du café, tout en étant centrée sur ses dialogues intérieurs concernant la deuxième commande, dans la mesure où l'annonce du cuisinier est extrêmement pertinente pour sa propre activité.

\section{a) distraction de la tâche principale}

L'attente de la première commande était d'abord une activité secondaire qui n'exigeait pas l'attention requise par la préparation de la deuxième commande ; dès que la serveuse entend le message du cuisinier, elle change rapidement la focalisation de son attention, suspend l'attention donnée à la deuxième commande et abandonne sa tâche principale en cours de réalisation (prendre les gâteaux pour les apporter aux clients).

\section{b) réaction mentale}

Le changement de la charge mentale survient plus précisément lorsque la serveuse réagit mentalement en pensant à sa commande qui est prête et au fait qu'elle doit l'apporter au client ("il y a un déclic qui se déclenche dans ma tête qui est l'autre commande à passer en premier, j'ai posé mes gâteaux et puis c'est euh l'assiette qui part en premier").

\section{c) libération et conscience de l'environnement immédiat}

Ensuite la serveuse ressent un sentiment de libération lorsque, en focalisant moins son attention sur la deuxième commande, elle est capable d'écouter à nouveau ce que ses collègues sont en train de dire. La charge mentale due aux dialogues internes pour la planification de l'activité privait la serveuse de la conscience de ce qui se passe alentour. Juste après avoir appris que sa commande est prête, elle peut participer à nouveau aux échanges ordinaires et aux blagues des collègues. Le sentiment de libération correspond à une plus grande disponibilité et par conséquent à une plus grande réceptivité. Le fait d'arrêter de penser à la commande en attente rend l'esprit de la serveuse «moins lourd » comme elle affirme dans l'entretien; elle a « plus de place dans sa mémoire » pour percevoir la situation de travail environnante, les actions des collègues, leurs blagues et autres échanges langagiers. ("mon esprit se libère d'une commande, donc j'ai l'impression que c'est moins lourd à porter, que ça y est, je peux écouter les autres trucs quoi").

Le fait que la conscience de la situation dépende de la charge mentale du sujet a déjà été souligné par Endsley (1995). Selon cet auteur, la conscience de la situation peut être restreinte par une attention limitée et une capacité de mémoire de travail, et elle dépend également de la charge de travail et du stress. L'exemple de Sophie montre clairement comment la conscience périphérique de ce qui se 
passe autour varie selon le degré d'engagement des acteurs dans une activité particulière, et il montre comment les acteurs peuvent avoir cette conscience ouverte et faible de l'environnement lorsque leur attention n'est pas trop focalisée sur un problème particulier.

L'engagement changeant des acteurs dans des activités particulières implique des compétences variables dans la prise de conscience des événements périphériques. L'observation sur le terrain est cruciale pour la conception de systèmes qui assistent le travail coopératif dans la mesure où, si les événements environnants sont rendus visibles et audibles par une technologie particulière, l'acteur, absorbé par son activité individuelle, peut momentanément être incapable de les percevoir.

\subsection{1.- Filtrage des objets et des personnes pertinents pour l'activité}

L'exemple de Sophie en attente de l'annonce orale proférée par le cuisinier montre bien que, quand elle est très engagée dans une activité, la serveuse n'entend - du bruit de fond provoqué par le café que ce qui est pertinent à son activité. L'exemple suivant montre comment les serveuses ont conscience des actions des autres dans la mesure où celles-ci sont pertinentes à l'accomplissement de leurs propres tâches. La perception visuelle est ici guidée par la recherche d'un plateau libre, et seuls les éléments de l'environnement qui sont pertinents à ce but sont remarqués.

Nous allons donc citer un autre extrait tiré d'un entretien d'explicitation mené avec une autre serveuse (Séverine). Cet extrait révèle les compétences particulières de cette serveuse expérimentée qui balaye visuellement l'espace de travail en remarquant ainsi les objets et les actions des collègues qui sont pertinents pour son activité. Le contexte de l'action décrit par Séverine est le suivant : elle est en train de revenir de la terrasse et a besoin d'un plateau vide pour préparer une nouvelle commande. Tout au long de son parcours d'action, elle accomplit de rapides contrôles visuels afin de trouver un plateau libre :

S - “(...) quand je revenais de la salle, je devais avoir une commande donc en même temps je cherchais un plateau de libre pour moi, je voyais qu'Olga était en train de débarasser un plateau, pour voir quel plateau je pouvais prendre pour pouvoir préparer ma commande (...)

I - donc, dans ce cas-là, tu regardes, tu regardes quoi, tu regardes Olga, tu regardes les plateaux, tu regardes le comptoir, tu vois?

S - je regarde tout en même temps (...) c'est rapide quand en fin de compte tout en avançant on regarde un peu derrière nous (...) pour essayer de trouver un plateau de libre pour ma prochaine commande.

I - là qu'est-ce que tu as vu quand tu as regardé ?

S - j'ai vu Olga qui était en train de débarasser les plateaux (...) il y en avait un que Sophie avait préparé sa commande dessus.

I - et là tu le vois comment euh? (en ralentissant le débit de parole)

S - parce qu'il était à côté du sucrier (...) donc Sophie avait préparé son plateau (...) mais j'étais pas seule à trouver, à essayer de trouver un plateau parce que je crois que Claude en cherchait aussi $(\ldots)$

I - (...) il était où Claude ?

S - il était derrière le bar, à côté de la machine à café.

I - alors on va essayer de voir ce que t'as vu dans, dans ce coup d'oeil quand t'arrives.

$\mathrm{S}$ - j'ai vu beaucoup de choses (rires)

I - donc tu regardes quoi, tu regardes...

S - vers Olga

I - vers Olga et tu regardes quoi d'Olga? 
S - euh le bar (...) donc son coin, son coin était plein de verres et tout ça qu'elle avait débarassés des plateaux, et j'allais, j'allais prendre le plateau pour prendre ma commande (... ) j'ai mis mon carnet de commande dessus.

I - et quand est-ce que tu t'es aperçue que Claude aussi était en train de de chercher un plateau (rires)

S - parce qu'il était en train de faire des cafés, il avait posé des cafés et euh lui avait tourné la tête pour voir ce qu'Olga faisait pour

I - et quand, est-ce que t'as vu les cafés, t'as vu les cafés, t'as vu les cafés avant Claude? t'as vu Claude avant ? tu vois

$\mathrm{S}$ - (soupire ; réfléchit) Claude il avait les, les tasses dans les mains (ralentissement du débit de parole) et quand je l'ai vu, il était en train de les poser

I - hm donc t'as vu ça et donc tu t'es dit qu'il allait chercher un plateau (...)

$\mathrm{S}$ - ben parce que bon il a posé ses tasses et après il a, il a regardé vers Olga donc, ma déduction à moi c'est que si, si il cherchait quelque chose quoi (...)

I - tu l'avais déjà vu le plateau que tu voulais ?

S - (...) ils étaient tous les deux libres, après quand moi je suis passée derrière le bar, Claude avait récupéré un plateau et moi j'avais récupéré un deuxième parce qu'il était libre pendant, parce qu'Olga l'avait presque fini, moi quand je suis arrivée (...) c'est rapide ouais c'est rapide, en tournant la tête on arrive à voir des choses (...) on voit l'une après l'autre quoi (...) tout en tournant la tête on arrive à voir, ça serait presque un film.

I - oui et dans ce film euh, tu vois, des objets tu les vois plus que d'autres ? ou le mouvement tu les vois plus que d'autres? ou c'est un ensemble?

S - (...) il y a des choses qu'on distingue plus que d'autres quoi, je vois euh, j'avais vu que Claude avait des tasses dans les mains donc euh (...)

I - hmm donc tu vois, là, si tu te remets comme ça dans la situation, tu vois Claude avec les deux cafés? ou tu vois seulement les deux cafés? ou tu vois seulement les mains, tu vois ? (...)

$\mathrm{S}$ - j'ai vu (ralentissement du débit de parole) j'ai pas vu Claude, la tête de Claude mais j'ai vu les mains de Claude avec les deux cafés en train de les passer, de les poser sur les sous-tasses (...)"

La serveuse expérimentée tout au long de son parcours d'action est en train de balayer visuellement l'environnement de travail afin de repérer les objets et les actions des collègues qui sont pertinents à l'accomplissement de sa propre tâche. Ses coups d'oeil sont orientés par la tâche particulière de la recherche d'un plateau libre et par la familiarité avec l'organisation spatiale du lieu de travail. Lorsque les caractéristiques de l'environnement sont en quelque sorte inspectées, elles sont tour à tour « ignorées » jusqu'au moment où l'information pertinente est découverte (Heath, \& Hindmarsch, $2000)^{3}$. Séverine lance des coups d'oeil vers des endroits particuliers autour du comptoir, là où elle sait que certaines actions sont accomplies par les collègues : la préparation du plateau près du sucrier, le lavage et l'essuyage d'Olga, les cafés qui vont être posés sur un plateau au bout du comptoir du bar. La familiarité avec la manière conventionnelle de faire les choses dans cette situation de travail fournit des actions prévisibles même si Séverine maîtrise également des problèmes imprévus qui exigent une solution improvisée sur le moment, et qui vont transformer son action selon les activités accomplies simultanément par les collègues. La serveuse a conscience de ce que les autres sont en train de faire, afin d'anticiper leurs interférences avec l'accomplissement de sa propre tâche, afin de savoir s'ils peuvent l'aider ou la déranger. La contrainte de partager le même espace de travail et les mêmes artefacts — ici les plateaux — oblige Séverine à contrôler visuellement les actions réalisées en parallèle par les coéquipiers, tout en formulant des hypothèses sur ce qu'ils sont en train de

3. "As successives features of the environnement are inspected, they are in turn "ignored" until the relevant information is discovered" (Heath, \& Hindmarsch 2000). 
faire. Pendant qu'elle lance des coups d'oeil dans l'espace de travail, la serveuse oriente son attention sur des actions et objets particuliers qui semblent émerger de l'arrière-plan et qui, temporairement, deviennent le premier foyer d'attention de la serveuse. Ces «objets clé » lui fournissent l'information pour trouver rapidement un plateau libre en anticipant comment les collègues vont déranger ou contribuer à sa recherche. Un plateau avec une commande posée dessus appartient à la serveuse qui est justement en train de le préparer et n'est donc pas disponible ; le coin d'Olga plein de verres signifie qu'elle est en train de débarasser un plateau qui va bientôt être libre; les mains de Claude avec des tasses à café indique que lui aussi va avoir besoin d'un plateau pour y poser les tasses. Tous ces objets ont du sens et sont pertinents pour Séverine, son attention est guidée par le sens pertinent de ces actions et de ces objets par rapport au but de trouver un plateau. Ce n'est pas tellement le caractère saillant de ces éléments perçus mais plutôt leur pertinence par rapport à la tâche qui guide ici le balayage visuel de la serveuse. Heath et Hindmarsch (2000) dans leur étude sur la collaboration accomplie par les coéquipiers dans une salle de contrôle du métro londonien, montrent en effet comment les objets représentent une ressource pour l'identification des actions et des activités des autres.

Tout acteur qui est engagé dans une activité de travail, n'a pas conscience au même niveau de tout ce qui se trouve dans l'environnement immédiat. Un filtrage est opéré et la plupart du temps celui-ci repose sur l'activité du sujet et sur sa connaissance des pratiques de travail accomplies par les membres d'une même équipe. Nous ne sommes pas d'accord avec Klein (1995) qui défend l'idée selon laquelle il faut étudier la conscience de la situation seulement dans le contexte de prises de décision en cas d'incidents, sinon les chercheurs risqueraient de conduire une recherche sans fin puisque la conscience de la situation (situation awareness) pourrait ainsi comprendre tout ce dont la personne a ou pourrait avoir conscience. Selon nous, c'est un risque seulement si l'on ne distingue pas les différents niveaux de conscience (awareness), et dans l'exemple précédent, qui illustre une situation ordinaire de travail, on voit que même s'il ne s'agit pas d'une prise de décision en cas d'incidents, les personnes n'ont pas conscience de tout au même niveau, et qu'elles changent très rapidement la focalisation de leur attention vers les éléments de l'environnement qui sont pertinents à l'activité dans laquelle elles sont engagées.

Selon Endsley (1995), la conscience de la situation repose sur la perception dans l'environnement d'éléments pertinents. Plus particulièrement, cette conscience est composée de la perception d'éléments dans l'environnement, de la compréhension de leur sens à la lumière des buts poursuivis par l'opérateur, et de la projection de leur état dans le futur proche. Ce cadre théorique proposé par Endsley doit tout de même être adopté avec précaution quant à la séquentialité du phénomène cognitif décrit. Flach (1995) souligne justement le risque de considérer la conscience de la situation comme une autre boîte dans le schéma du traitement de l'information, située entre l'attention et la prise de décision. Il préfère la traiter comme un concept qui donne la priorité à l'interaction entre l'homme et l'environnement, plutôt que comme une phase du système de traitement de l'information : "Situation awareness is a phenomenon that challenges the reductionist assumptions that permitted parsing of the information-processing system into indenpendant stages: Situation awareness reflects the need for a more holistic approach to the human performance".

\section{3.- Conclusion}

Nous espérons contribuer par cet article aux études de terrain sur le travail, et offrir une description détaillée des pratiques quotidiennement mises en œuvre par les acteurs pour la coordination de leurs actions et l'utilisation de lieux et artefacts partagés. En étudiant la conscience périphérique (awareness) dans une situation de travail, sont prises en considération non seulement les activités centrales mais tous les autres canaux qui, à différents niveaux de conscience, contribuent à la coordination des agents, comme l'ont montré précédemment Heath et Luff (1996) dans le cadre des salles de contrôle 
du métro, en soulignant le rôle de la vision et de l'audition périphérique pour la coordination au travail.

Nous avons montré que l' «awareness » est une notion mal définie qui inclut de nombreux processus différents selon les auteurs et qu'il faut surtout distinguer différents degrés de conscience périphérique qui dépendent du niveau de réfléchissement que le sujet a de son activité et de son foyer attentionnel.

Cette variété de degrés rend la notion intéressante en ce qu'elle rend compte non seulement d'activités conscientes et aisément accessibles, mais aussi de la possibilité d'analyser la partie dans l'ombre, implicite et floue des pratiques de travail coopératif, même celles qui sont pré-réfléchies, inscrites dans l'action et plus complexes à verbaliser.

Dans la situation du café-restaurant, dans le cas présenté par Sophie, on peut distinguer différents niveaux de conscience selon les activités : (a) d'un niveau de conscience réfléchie élevé pour l'activité principale, comme préparer une commande qui est une activité assez conceptualisée par les agents experts comme Sophie, avec la possibilité de décrire les buts, prérequis, moyens, ... (b) à un niveau de conscience moyen pour certains processus de communication (entre le cuisinier et les serveurs par exemple) et pour le moyen visuel d'utiliser les parcours d'action des collègues ; (c) à un niveau de faible conscience périphérique concernant la synchronisation des mouvements pour éviter les collisions ; ce sont des activités pré-réfléchies qui ne sont pas facilement décrites par les acteurs.

Ces niveaux ne sont bien sûr pas strictement attachés à telle ou telle activité mais peuvent varier selon le contexte, telle activité devenant centrale et consciente par instants et repassant au second plan à d'autres. Il est en effet important de souligner que, durant l'activité de travail, la conscience périphérique de différents collègues et objets varie constamment. Après avoir été très conscient des actions de ses collègues, un serveur peut soudain en devenir très peu conscient parce qu'il est impliqué dans une activité particulière ou parce que l'environnement a changé. L'attention périphérique peut soudain être restreinte ou élargie par la nature de l'activité qui évolue, nous avons observé ce phénomène grâce à l'entretien d'explicitation de Sophie.

La conscience périphérique est donc caractérisée par la discontinuité et les variations dynamiques. Les foyers attentionnels simultanés sont nombreux, les acteurs sont conscients de beaucoup de choses à la fois et les niveaux de conscience changent constamment : un objet qui était au centre de l'attention passe rapidement à l'arrière-plan, selon les changements de l'environnement et l'évolution de l'activité.

Nos données indiquent également que le niveau de conscience est dépendant de la charge cognitive d'un acteur : on a vu comment Sophie a beaucoup à penser par moments et ne peut alors rester consciente de ce qui se passe autour d'elle ; les serveurs sont souvent conscients de ce qui est le plus pertinent pour gérer au mieux leur activité, on l'observe également dans le cas du plateau de Séverine.

La plurisensorialité impliquée pour la conscience périphérique doit être soulignée : non seulement la perception visuelle et auditive mais aussi la perception kinesthésique sont actives pour la synchronisation collective et l'évitement des collusions dans l'espace restreint où les serveurs se croisent sans cesse.

Nos données indiquent également comment une légère conscience périphérique des collègues du café est à la fois nécessaire et risquée. Elle est nécessaire et utile parce que les modes d'articulation sont légers et peu intrusifs et rendent possible les activités parallèles, ce qui est important pour une telle situation où le temps et l'espace sont restreints. Elle a l'avantage de permettre des allocations de tâche émergentes et opportunistes et de créer ainsi un système ouvert, flexible et adaptable. Cependant ces modes de coordination ont aussi le désavantage d'être incertains et moins fiables, essentiellement parce qu'ils sont implicites et parce que leur caractère faiblement intrusif amène moins de feedbacks et de régulateurs : le cuisinier n'interrompt pas sa tâche de confection des sand- 
wichs pour donner un feedback réactif à la demande des serveurs mais les serveurs ne sont pas assurés qu'il les a bien entendus.

Pour la conception de systèmes facilitant la conscience périphérique, le besoin des acteurs de rester sensibles aux actions des collègues environnants doit être précautionneusement contrebalancé avec le risque de les submerger avec trop d'informations qu'ils ne peuvent traiter (mais que leurs collègues pensent qu'ils peuvent traiter).

La dynamique sociale et psychologique sont les deux faces du travail coopératif (Cahour, \& Pemberton, 2001); l'analyse de l'émergence sociale des arrangements collectifs, et de la gestion cognitive individuelle de la situation de travail sont des points de vue différents et complémentaires sur une même situation, de même que les méthodologies pour les approcher (observation et interviews) sont différentes.

Nous avons montré qu'il est possible d'amener les acteurs à verbaliser des activités pré-réfléchies telles que les divers foyers attentionnels qu'ils gèrent simultanément pour remplir les tâches individuelles et collectives (les commandes de Sophie), ou comme l'attention qui est orientée sélectivement vers certains objets, actions ou personnes parce qu'ils sont pertinents par rapport au cours d'action (le plateau de Séverine). Pour cela il est nécessaire cependant d'utiliser des techniques de recueil de données qui ont été conçues spécifiquement pour obtenir des descriptions de moments vécus particuliers, et non pas des descriptions générales sur l'activité. Cela apparaît être une méthodologie de recherche fructueuse, qui soulève des questions mais qui permet certainement d'aller plus loin dans l'analyse des processus cognitifs en jeu lors de situations de travail collectif. Par exemple, pour la conception de lieux de travail au niveau artefactuel et organisationnel, il est crucial d'identifier quels sont les objets pertinents dans l'environnement pour les acteurs, et l'entretien d'explicitation le permet. Il est également important de pouvoir savoir quand un acteur a suffisamment de disponibilité cognitive pour être conscient d'un événement. Lorsqu'on s'intéresse à ce type d'information sur le travail, l'analyse vidéo de la situation et les techniques d'interviews classiques ne sont plus suffisants. Nous avons vu comment les serveuses, même si elles ont momentanément un plan qu'elles tentent de suivre, réagissent de façon très flexible aux contingences de la situation (localisation des objets, actions et parcours d'action des collègues). Leur attention et parcours d'action sont sans cesse en train d'émerger d'un contexte situationnel en constante évolution. La technique de questionnement doit donc suivre pas à pas ce qui s'est passé pour l'acteur en situation.

La façon opportuniste et flexible dont les acteurs adaptent leurs actions aux contingences de la situation immédiate a été soulignée par Suchman (1987) et par Lave (1988); cet auteur critique l'approche cognitive traditionnelle qui surestime selon lui le rôle des plans et des représentations mentales comme déterminant la conduite individuelle. La description détaillée de l'activité dans une épicerie (Lave, 1988) met en exergue la nature improvisatrice de l'activité humaine en fonction des contingences d'une situation donnée. Ces études empiriques qui décrivent finement le séquencement temporel des actions en cours indiquent que l'organisation de l'action située est une propriété émergente de l'interaction qui se déploie dynamiquement entre les acteurs, et entre les acteurs et l'environnement de leurs actions (Suchman, 1987 ; Lave, 1988 ; Nardi, 1996).

Enfin, il faut noter que la conscience périphérique de l'environnement humain et artefactuel a une fonction opérationnelle mais aussi interpersonnelle : elle est utile pour une allocation flexible des tâches et pour l'articulation efficace du travail mais elle est également nécessaire pour les relations sociales du groupe de collègues et pour la cohésion interne de ce groupe.

\section{REMERCIEMENTS}

Nous remercions nos collègues du réseau européen TMR «COTCOS » pour les discussions fructueuses que nous avons eu lors des réunions du groupe de travail sur "awareness and cooperative work" (et 
particulièrement Bernard Gardin). Merci aussi à Pierre Vermersch pour les échanges sur son approche des processus attentionnels et sa méthode d'entretien.

\section{RÉFÉRENCES}

Benford, S., \& Fahlen, L.E. (1993). A spatial model of interaction in large virtual environments. Proceedings of Third European Conference on CSCW, ECSCW'93, Milano, Italy (pp. 106-124). Kluwer Academic Press.

Cahour, B., \& Pemberton, L. (2001). A model of conversational positioning in collaborative design dialogues. AI and society, 15(4), 344-358.

Clark, H.H. (1996). Using Language. Cambridge: Cambridge University Press.

Clark, H.H., \& Brennan, S.E. (1991). Grounding in conversation. In L.B. Resnick, J.M. Levine, \& S.D. Teasley (Eds.), Perspective on socially shared cognition (pp. 127-149). Washington D.C.: American Psychological Association.

Dix, A. (1997). Challenges for cooperative work on the WEB: An analytical approach. CSCW Journal (Kluwer), 7 (2-3), 135-156.

Endsley, M.R.(1995). Towards a theory of situation awareness in dynamic systems. Human Factors, 37 (1), 32-64.

Ericsson, K.A., \& Simon, H.A. (1984/1993). Protocol analysis, verbal protocols as data. Cambridge, MA: MIT Press.

Flach, J.M. (1995). Situation awareness: proceed with caution. Human Factors, 37 (1), 149-157.

Goffman, E. (1973). La mise en scène de la vie quotidienne : les relations en public. Paris : Editions de Minuit (traduction française).

Goffman, E. (1974). Frame analysis. New York: Harper Row.

Heath, C. \& Hindmarsh, J. (2000). Configuring objects in action: From mutual space to media space. Mind, culture and activity, 7 (1/2).

Heath, C., Jirotka, M., Luff, P., \& Hindmarsh J. (1995). Unpacking collaboration : the interactional organisation of trading in a city dealing room. CSCW Journal, 3 (2), 147-165.

Heath, C., \& Luff, P. (1994) Activité distribuée et organisation de l'interaction. Sociologie du Travail XXXVI (4), 523-545.

Heath, C., \& Luff, P. (1996). Convergent activities : collaborative work and multimedia technology in London underground line control rooms. In D. Middleton, \& Y. Engestrom (Eds), Cognition and communication at work (pp. 96-130). Cambridge: Cambridge University Press.

Heath, C., \& Luff, P. (2000). Technology in action. Cambridge: Cambridge University Press.

Husserl, E. (1950). Idées directrices pour une phénoménologie. Paris: Gallimard (traduction française.

Husserl, E. (1970). Expérience et Jugement. Paris: PUF (traduction française. de 1954).

Joseph, I. (1994), Attention distribuée et attention focalisée, Les protocoles de la coopération au PCC de la ligne A du RER. Sociologie du Travail XXXVI (4), 563-585

Klein, G. (1995). Studying situation awareness in the context of decision-making incidents. In D.J. Garland, \& M.R. Endsley (Eds.), Proceedings of the International Conference on Experimental Analysis and Measurement of Situation Awareness (pp. 177-181). Daytona beach, Florida: Embry-Riddle Aeronautical University Press.

Lave, J. (1988). Cognition in practice. Cambridge: Cambridge University Press.

Mark, G., Fuchs, L., \& Sohlenkamp, M. (1997). Supporting groupware conventions through contextual awareness. In J. Hughes et al. (Eds.), Proceedings ECSCW'97, Lancaster (pp. 253-268). Kluwer.

Nardi, B. (1996). Studying context : a comparison of activity theory, situated action models and distributed cognition. In B. Nardi (Ed), Context and consciousness (pp. 69-102). Cambridge: the MIT Press.

O'Day, V., Bobrow, D., Bobrow, K., Shirley, M., Hughes, B. \& Walters, J. (1998). Moving practice: from classrooms to MOO rooms. CSCW Journal (Kluwer), 7 (1-2), 9-45. 
Piaget, J. (1974). La prise de conscience. Paris: PUF.

Richard, J.F. (1980). L'attention. Paris:PUF.

Rodden, T. (1996). Populating the application: a model of awareness for cooperative applications. In M.S. Ackrman (Ed.), Proceedings CSCW'96 Conference, (pp. 87-96). Boston.

Sandor, O., Bogdan, C., \& Bowers, J. (1997). Aether: an awareness engine for CSCW. In J. Hughes et al. (Eds.), Proceedings ECSCW'97, Lancaster (pp. 221-236). Kluwer.

Schmidt, K. (2000). Managing the complexity of cooperative work. In Modelling and Complexity, Deliverable WP3 of COTCOS EU TMR network FMRX-CT96-0014.

Sperber, D. \& Wilson, D. (1986). Relevance ; communication and cognition. Oxford: Blackwell.

Stiemerling, O. \& Wulf ,V. (1998). Beyond "yes or no" - extending access control in groupware with awareness and negociation, In Proceedings COOP'98, International conference on the design of cooperative systems, Cannes, INRIA Press.

Suchman, L.A. (1987). Plans and situated actions. Cambridge: Cambridge University Press.

Sudnov, D. (1972). Temporal parameters of interpersonal observation. In D. Sudnov (Ed.), Studies in social interaction (pp. 259-279). New-York: Free Press.

Theureau J. (1992). Le cours d'action: analyse sémiologique. Essai d'une anthropologie cognitive située. Berne: Peter Lang.

Vermersch, P. (1994). L'entretien d'explicitation. Paris: ESF.

Vermersch, P. (1999). Introspection as practice. Journal of Consciousness Studies, 6 (2-3), 17-42.

\section{RÉFÉRENCEMENT}

Cahour, B. , \& Pentimalli, B. (2005). Conscience périphérique et travail coopératif dans un café-restaurant @ ctivités, 2 (1), 50-75, http://www.activites.org/v2n1/cahour.pdf

\section{RESUMEN}

Consciencia periférica y trabajo cooperativo en un café-restaurante.

En este artículo presentamos un estudio de campo de las actividades de cooperación en un café-restaurante entre camareros y cocineros. En especial, mostramos cómo el nivel de conciencia periférica (awareness) de lo que sucede en el entorno inmediato de trabajo, varía continuamente y se encuentra ligado a los mecanismos de atención de los participantes. En primera instancia, y a partir de algunas secuencias de video, este artículo analiza las funciones de la conciencia periférica entre compañeros de equipo gracias a la escucha flotante, la visión periférica y las percepciones kinestésicas : su necesidad de realizar desplazamientos cortos y comunicarse rápidamente para ganar tiempo, su necesidad de evitar colisiones en un espacio estrecho, etc...

En segunda instancia, y gracias a las entrevistas de explicitación realizadas con dos camareras, presentamos un análisis detallado de las variaciones de la atención que se encuentran ligadas a la sobrecarga cognitiva de la actividad en curso de realización, y a los objetos y personas pertinentes a la actividad misma.

\section{PALABRAS CLAVE}

actividades de cooperación, niveles de conciencia periférica, focalización y grados de atención, percepciones kinestésicas, comunicación, dinámicas socio-cognitivas, pluri-sensorialidad. 


\section{RÉSUMÉ}

Dans cet article nous présentons une étude de terrain des activités de coopération qui ont lieu dans un café-restaurant entre serveurs et cuisiniers. Plus particulièrement, nous montrons comment le niveau de conscience périphérique (awareness) de ce qui se passe dans l'environnement immédiat de travail varie continuellement et est lié aux mécanismes d'attention des participants. Dans un premier temps, à partir de quelques séquences vidéo, cet article analyse les fonctions de la conscience périphérique entre coéquipiers grâce à l'écoute flottante, la vision périphérique et les perceptions kinesthésiques : leur besoin d'accomplir de courts déplacements et de communiquer rapidement pour gagner du temps, leur besoin d'éviter des collisions dans un espace étroit, etc.

Dans un deuxième temps, grâce aux entretiens d'explicitation menés avec deux serveuses, nous présentons une analyse détaillée des variations de l'attention qui sont liées à la surcharge cognitive de l'activité en cours de réalisation, et aux objets et personnes pertinents pour cette même activité.

MOTS CLÉ :

Activités de coopération, niveaux de conscience périphérique, focalisations et degrés de l'attention, perceptions kinesthésiques, communication, dynamiques socio-cognitives, pluri-sensorialité. 